\title{
Diffuse panbronchiolitis
}

\section{Sheng-Hsiang Lin MD, Yu-Shu Liao DDS}

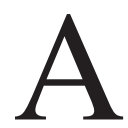

63-year-old man presented with a 10-year history of cough with copious purulent sputum and progressive exertional shortness of breath. His medical history was remarkable only for chronic sinusitis. Coarse crackles and rhonchi were heard bilaterally over the lower lung fields. Laboratory investigations including complete blood count, biochemistry, and sputum cultures for mycobacteria and fungus were normal. Chest radiography showed diffuse reticulonodular shadows bilaterally with a lower-lung predominance, and high-resolution computed tomography (CT) showed bronchiectasis, centrilobular nodules and tree-in-bud opacities bilaterally (Figure 1). Diffuse panbronchiolitis was diagnosed.

After treatment with oral erythromycin for six months, our patient's symptoms markedly improved. The small nodular shadows on chest radiography disappeared, and there was partial resolution of centrilobular nodules on high-resolution CT (Appendix 1, available at www.cmaj.ca/lookup /suppl/doi:10.1503/cmaj.111671/-/DC1).

Diffuse panbronchiolitis is characterized by chronic sinobronchial inflammation and is seen primarily in Asian populations. ${ }^{1,2}$ Its prevalence in Japan has been reported at $1 / 10000$; it has also been described in North America. ${ }^{1,3,4}$ Productive cough, shortness of breath, coarse lung crackles and a history of sinusitis are noted in the vast majority of patients. ${ }^{1}$ Chest radiography typically shows diffuse small nodular shadows; high-resolution $\mathrm{CT}$ is very useful in identifying the characteristic lesions, including diffuse centrilobular nodules, tree-in-bud opacities, bronchiolectasis and bronchiectasis. ${ }^{1}$

Diffuse panbronchiolitis had a poor prognosis before the use of erythromycin. Long-term and low-dose treatment with erythromycin has been shown to improve pulmonary function, clinical symptoms and survival rate in these patients, perhaps because of its anti-inflammatory proper-
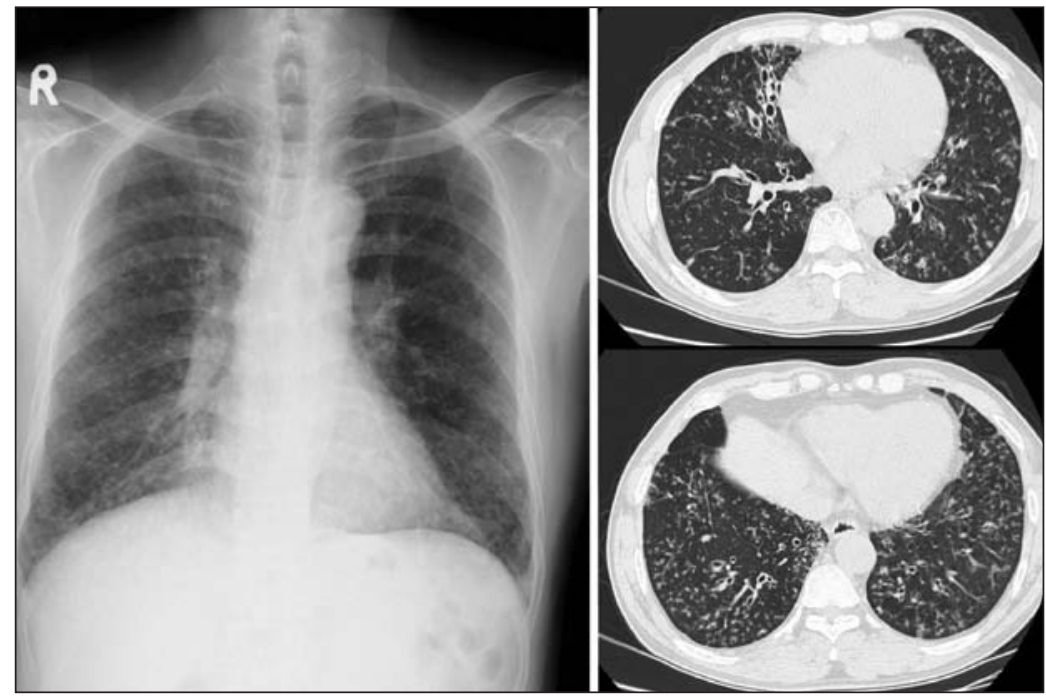

Figure 1: Chest radiograph showing diffuse reticulonodular shadows, and highresolution computed tomographic scan showing bronchiectasis, centrilobular nodules and tree-in-bud opacities in a 63-year-old man with a 10-year history of cough, sputum production and dyspnea.

ties. ${ }^{1,2}$ However, in its advanced stages, diffuse panbronchiolitis is characterized by extensive bronchiectasis and carries a poor prognosis despite treatment with erythromycin. ${ }^{2}$ Recognizing the characteristic radiographic findings of this condition is important for the diagnosis and early start of treatment with macrolides. ${ }^{5}$

\section{References}

1. Azuma A, Kudoh S. Diffuse panbronchiolitis in East Asia. Respirology 2006;11:249-61.

2. Kudoh S, Azuma A, Yamamoto M, et al. Improvement of survival in patients with diffuse panbronchiolitis treated with lowdose erythromycin. Am J Respir Crit Care Med 1998;157: 1829-32.

3. Sandrini A, Balter MS, Chapman KR. Diffuse panbronchiolitis in a Caucasian man in Canada. Can Respir J 2003;10:449-51.

4. Krishnan P, Thachil R, Gillego V. Diffuse panbronchiolitis: a treatable sinobronchial disease in need of recognition in the United States. Chest 2002;121:659-61.

5. Poletti V, Casoni G, Chilosi M, et al. Diffuse panbronchiolitis. Eur Respir J 2006;28:862-71.
Competing interests: None declared.

This article has been peer reviewed.

Affiliations: From the Division of Pulmonary Medicine (Lin), Department of Internal Medicine, New Taipei City Hospital, New Taipei City, Taiwan; and the Department of Prosthodontics (Liao), Chang-Gung Memorial Hospital, Taipei, Taiwan

Correspondence: Dr. Sheng-Hsiang Lin, linsh01@gmail.com

CMAJ 2012. DOI:10.1503 /cmaj.111671 